were compared with corresponding findings on retrograde urethrogram/voiding cystourethrogram to determine the ability of uroflowmetry to predict recurrence.

Results: A total of 278 men (68\%) met study inclusion criteria, of whom $63(23 \%)$ had recurrent stricture. Using a maximum flow rate of less than $10 \mathrm{~mL}$ per second resulted in only $54 \%$ test sensitivity to predict recurrence. The highest sensitivity and negative predictive value (each 99\%) were achieved when all men with symptoms and/or obstructed flow curves were evaluated. Symptoms alone had a high specificity (87\%), sensitivity (88\%) and negative predictive value $(95 \%)$.

Conclusions: Uroflowmetry is an adequate test to screen for postoperative stricture recurrence but only when the voiding curve and urinary symptoms are also evaluated. The flow rate alone does not appear to be a reliable tool to evaluate stricture recurrence.

doi: $10.1590 / S 1677-55382010000500023$

\title{
A simplified protocol for evaluating and monitoring urethral stricture patients minimizes cost without compromising patient outcome
}

Okorie CO, Pisters LL, Ndasi HT, Fekadu A

Pan-African Academy of Christian Surgeons at Banso Baptist Hospital, Kumbo, NWP, Cameroon

Trop Doct. 2010; 40: 134-7

Abstract: Uroflowmetry, urethrocystoscopy and urethrography are either not readily available or the cost is prohibitive for many patients in low-resource countries. This paper examines the use of clinical history in post-urethroplasty follow-up. We retrospectively reviewed the outcome of 54 post-urethroplasty patients. Preoperative diagnostic work-up included simple blood tests and a retrograde urethrography, and postoperatively we did not perform any immediate diagnostic work-up. Follow-up of these patients was done through mobile phone calls and personal contacts. Eighty-nine per cent of our patients reported acceptable voiding over a mean follow-up period of 48.4 months $-79.6 \%$ were followed using mobile phone contact. In the majority of the urethral strictures cases, diagnostic work up can be kept to a minimum, thereby reducing cost. Follow-up can be done via phone calls and personal contact in many African countries where compliance is frequently less than encouraging. The spread of mobile phone networks across the continent has been remarkable.

\section{Editorial Comment}

The study by Erickson et al. seeks to arrive at a non-invasive means of predicting urethral stricture recurrence after urethroplasty. Uroflowmetry is attractive in that it is an objective test. The authors state that maximum urinary flow rate is not by itself a very good predictor of failure but that the combination of maximum flow rate, voiding pattern and urinary obstructive symptoms is predictive. Indeed, the combination of these three factors was the most predictive of a man having a recurrence (PPV 90\%); yet this combination misses half the strictures (sensitivity 51\%). This low sensitivity means few physicians would be inclined to forgo more invasive testing in any man. Although it was the goal of the authors to find a predictive tool, what would be more helpful to the patient would be a screening tool. A good screening tool helps a portion of the population avoid advanced confirmatory testing, while still preserving high sensitivity. With the latter goal in mind, a high threshold for maximum urinary flow rate $(<20 \mathrm{cc} / \mathrm{sec})$ has excellent sensitivity $(92 \%)$ and NPV (96\%), meaning that it captures $92 \%$ of strictures and only misses $4 \%$. While PPV is low (48\%) this is acceptable as a screening tool - it just means that a little more than half of men who undergo RUG/VCUG will have no recurrent stricture. At the same time, more than half of the population in this study could avoid a RUG/VCUG because their flow rates were over $20 \mathrm{cc} / \mathrm{sec}$ - this is the proper role of a screening tool. 
The goal of identifying a useful screening tool for urethral stricture recurrence is echoed in the second article, by Okorie et al. They describe using mobile phones to survey urinary symptoms after urethral stricture treatment. Although the symptoms reported over the telephone were not validated objectively, the concept of telephone follow-up is important, especially in areas of the world where travel to a referral center is difficult. Others have previously shown that home measurement of timed urinary flow correlates well with office-measured maximum urinary flow rate (1). If we were able to validate a means of screening patients over the telephone with timed urinary flow rates with or without telephone-administered validated questionnaires we could spare many patients the travel to a referral center for continued follow-up.

\title{
Reference
}

1. Bloom DA, Foster WD, McLeod DG, Mittemeyer BT, Stutzman RE: Cost-effective uroflowmetry in men. J Urol. 1985; 133: 421-4.

\author{
Dr. Sean P. Elliott \\ Department of Urology Surgery \\ University of Minnesota \\ Minneapolis, Minnesota, USA \\ E-mail: selliott@umn.edu
}

\section{UROLOGICAL ONCOLOGY}

doi: $10.1590 / S 1677-55382010000500024$

\section{Should bladder cuff excision remain the standard of care at nephroureterectomy in patients with urothelial carcinoma of the renal pelvis? A population-based study}

Lughezzani G, Sun M, Perrotte P, Shariat SF, Jeldres C, Budaus L, Alasker A, Duclos A, Widmer H, Latour M, Guazzoni G, Montorsi F, Karakiewicz PI

Cancer Prognosis and Health Outcomes Unit, University of Montreal Health Center, Montreal, Québec, Canada; Department of Urology, Vita-Salute San Raffaele University, Milan, Italy

Eur. Urol. 57: 956-962, 2010

Background: A large, multi-institutional, tertiary care center study suggested no benefit from bladder cuff excision (BCE) at nephroureterectomy in patients with upper tract urothelial carcinoma (UC).

Objective: We tested and quantified the prognostic impact of BCE at nephroureterectomy on cancer-specific mortality (CSM) in a large population-based cohort of patients with UC of the renal pelvis.

Design, Setting, And Participants: A cohort of 4210 patients with UC of the renal pelvis were treated with nephroureterectomy with (NUC) or without (NU) a BCE between 1988 and 2006 within 17 Surveillance, Epidemiology, and End Results registries.

Measurements: Cumulative incidence plots and competing risks regression models compared CSM after either NUC or NU. Covariates consisted of pathologic T and N stages, grade, age, year of surgery, gender, and race. Results and Limitations: Respectively, 2492 (59.2\%) and 1718 (40.8\%) patients underwent a nephroureterectomy with or without BCE. In univariable and multivariable analyses, BCE omission increased CSM rates in patients with $\mathrm{pT} 3 \mathrm{~N} 0 / \mathrm{x}, \mathrm{pT} 4 \mathrm{~N} 0 / \mathrm{x}$, and $\mathrm{pT}$ (any)N1-3 UC of the renal pelvis. For example, in patients with pT3N0/x disease, holding all other variables constant, BCE omission increased CSM in a 1.25-fold fashion 